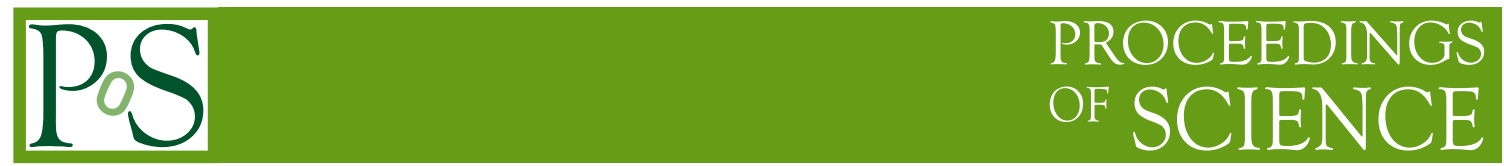

\title{
Non-relativistic EFT for hydrogen atom at finite temperature
}

\author{
Miguel Ángel Escobedo* \\ Departament d'Estructura i Constituents de la Matèria and \\ Institut de Ciències del Cosmos, Universitat de Barcelona \\ Diagonal 647, E-08028 Barcelona, Catalonia, Spain \\ E-mail: mesco@ecm.ub.es
}

Heavy Quarkonium dissociation in Heavy Ion Collisions is one of the more reliable tests of the formation of a Quark Gluon Plasma in these experiments. However, at the moment it is not theoretically clear what is the exact temperature in which this happens for the different Heavy Quarkonium states. In this talk, we use Hydrogen atom as a toy model for Heavy Quarkonium, making use of Non-relativistic EFT, that have proven to be very useful at $T=0$.

International Workshop on Effective Field Theories: from the pion to the upsilon 2-6 February 2009

Valencia, Spain

${ }^{*}$ Speaker. 


\section{Introduction}

Already in the 80's, it was conjectured that, if a Quark Gluon Plasma (QGP) was formed in Heavy Ions Collision, it should be signalled by a Heavy Quarkonium Suppression [1]. This suppression pattern have been observed both in SPS and RHIC [2,3] and is one of the more reliable tests of the production of a QGP in these experiments. However, interpretation of RHIC data is not so clear, because it was expected a bigger suppression than what was actually found. Also there is some tension between the dissociation temperatures that are predicted with different theoretical tools [4].

Recently, some attempts have been made of applying Non-Relativistic EFT for this problem [5, 6]. At zero temperature, these EFTs have proven to be very powerful [7] and now they are standard tools for Heavy Quarkonium. This EFTs are Non-relativistic QCD (NRQCD) [8] and potential NRQCD (pNRQCD) [9]. Historically, these theories were tested at the beginning also in QED problems [10], as a toy model for Heavy Quarkonium, showing that the use of these EFTs made computations of old known quantities (for example, the Lamb Shift) much easier.

Following this, we apply NRQED and pNRQED to the study of the spectroscopy of Hydrogen atom as a toy model for Heavy Quarkonium. In this proceedings there is also a talk by Nora Brambilla about Heavy Quarkonium at finite temperature in the static limit, which is complementary to this work [11].

Further details of what is discussed here can be found in [5].

\section{Needed theoretical tools}

In this section we review needed theoretical tools for the computations that we are going to make. Mainly, Non-relativistic EFTs and Thermal Field Theory.

In a Hydrogen atom there exist various scales that make very convenient to work with EFTs.

- The electron mass $m$ (we consider the proton to be infinitely heavy), which is the hard scale.

- The typical radius and typical tri-momentum, which are of order $m \alpha$, the soft scale.

- The binding energy, $m \alpha^{2}$, the ultrasoft scale.

\subsection{NRQED}

NRQED was derived in [8]. In this EFT the hard scale have been integrated out. In the fermion sector we go from bispinors to spinors and the photon sector is almost not modified.

$$
\begin{gathered}
\mathscr{L}=\psi^{\dagger}\left(i D^{0}+\frac{\vec{D}^{2}}{2 m}+\frac{\vec{D}^{4}}{8 m^{3}}+c_{F} e \frac{\vec{\sigma} \vec{B}}{2 m}+c_{D} e \frac{|\vec{\nabla} \vec{E}|}{8 m^{2}}+i c_{S} e \frac{\vec{\sigma}(\vec{D} \times \vec{E}-\vec{E} \times \vec{D})}{8 m^{2}}\right) \psi+ \\
+N^{\dagger} i D^{0} N-\frac{1}{4} d_{1} F_{\mu \nu} F^{\mu \nu}+\frac{d_{2}}{m^{2}} F_{\mu \nu} D^{2} F^{\mu \nu} .
\end{gathered}
$$

These are the terms that are needed for the computation of the spectroscopy up to order $m \alpha^{5}$. Notice that in addition to an expansion in $\alpha$ it is also an expansion in $\frac{1}{m}$. However, the power counting of this theory is not obvious because two different scales are not disentangled (the soft and the ultrasoft scales). 

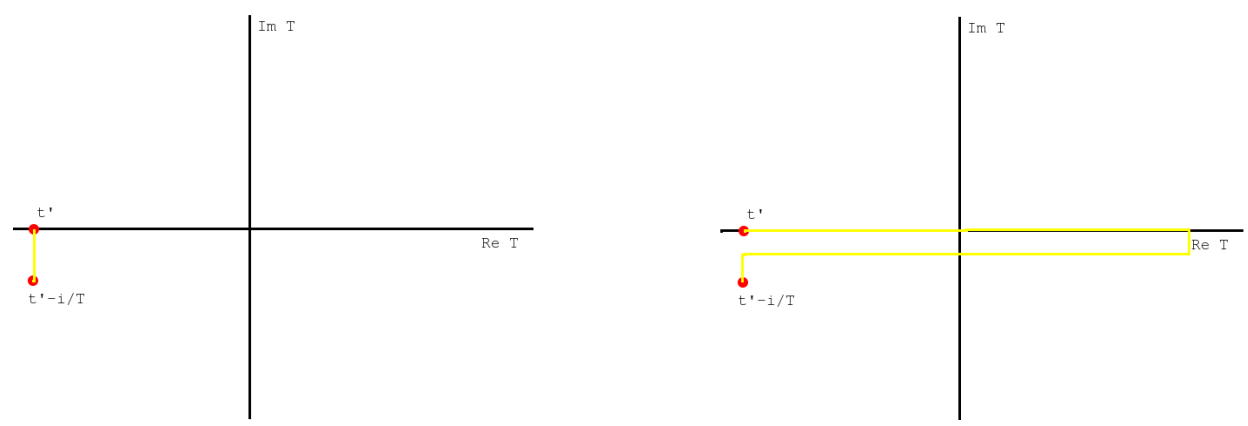

Figure 1: The left hand side is a representation of the integration path in the complex time plane of the imaginary time formalism. The right hand side is the same for the real time formalism.

\subsection{PNRQED}

This EFT was derived in [9]. It is the result of integrating out the soft scale in NRQED.

$$
\begin{gathered}
L_{p N R Q E D}=\int d^{3} x\left(\psi^{\dagger}\left\{i D^{0}+\frac{\vec{D}^{2}}{2 m}+\frac{\vec{D}^{4}}{8 m^{3}}\right\} \psi+N^{\dagger} i D^{0} N-\frac{1}{4} F_{\mu v} F^{\mu v}\right)+ \\
+\int d^{3} x_{1} d^{3} x_{2} N^{\dagger} N\left(t, x_{2}\right)\left(\frac{Z \alpha}{\left|x_{1}-x_{2}\right|}+\frac{Z e^{2}}{m^{2}}\left(-\frac{c_{D}}{8}+4 d_{2}\right) \delta^{3}\left(x_{1}-x_{2}\right)+\right. \\
\left.+i c_{S} \frac{Z \alpha}{4 m^{2}} \vec{\sigma}\left(\frac{\overrightarrow{x_{1}}-\overrightarrow{x_{2}}}{\left|x_{1}-x_{2}\right|^{3}} \times \vec{\nabla}\right)\right) \psi^{\dagger} \psi\left(t, x_{1}\right)
\end{gathered}
$$

Here, some of the Wilson coefficients are potentials. With this EFT it is easy to obtain the spectroscopy up to order $m \alpha^{5}$. In particular, the Lamb shift is much easier to calculate in this way than with Bethe-Salpeter formalism. This shift is of order $\delta E \sim m \alpha^{5} \log (E / m)$, and in its computation, a renormalization scale $\mu$ dependency that comes from a ultraviolet divergence in the ultrasoft scale is cancelled with another $\mu$ dependency in the Wilson coefficient of NRQED $c_{D}$.

\subsection{Thermal Field Theory}

What is known as Thermal Field Theory is Quantum Field Theory at finite temperature. Here we are going to make a very brief review, more details can be found in [12]. At finite temperature one is interested in computing the expected value of an operator in a thermal average instead of the vacuum.

$$
\langle A\rangle_{\beta}=\frac{1}{\log Z} \operatorname{Tr}\left(e^{-H \beta} A\right),
$$

where $\beta=1 / T$. $e^{-H \beta}$ is like a time evolution operator, but with an imaginary part. Combining this idea with the path integral formalism is the key point for Thermal Field Theory. There exist two formalism that are equivalent, but each one has its advantages depending on the situation.

- The imaginary time formalism.

This is the more suitable formalism for time-independent quantities. The path integral goes from one time to the same time with an imaginary component $-i / T$. This gives rise to a discretization of the possible frequencies in the momentum space Feynman rules. Dynamic quantities can be dealt if after the computation analytic continuation is done.

- The real time formalism. 


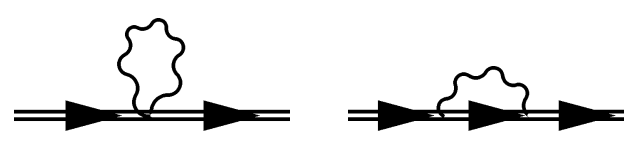

Figure 2: Double line represents the atom, and wavy lines the photon

This is the formalism that we use in this work. Implicitly, it is stated that the system was in thermal equilibrium in the far past $(t=-\infty)$ and that it will be in thermal equilibrium in the far future $(t=\infty)$. Because in this path we integrate for all times in the real axis, expectation values of operators in different times can be computed.

In the calculation of the partition function the vertical lines decouples from the horizontal ones. The fact that there are two horizontal lines gives rise to a doubling of degrees of freedom. The physical meaning of this is that there are particles from the system (type 1) and also particles of the thermal bath that interacts with it (type 2).

The length of the first vertical line can be chosen freely [12] between 0 to $1 / T$. Here we use the path with 0 length (Closed Time Formalism). As an example, we write the propagator for a scalar particle in this formalism.

$$
\begin{gathered}
\Lambda(K)=\left(\begin{array}{ll}
\Lambda_{11} & \Lambda_{12} \\
\Lambda_{21} & \Lambda_{22}
\end{array}\right) \\
\Lambda_{11}=\frac{i}{K^{2}-m^{2}+i \varepsilon}+2 \pi \delta\left(K^{2}-m^{2}\right) n_{B}\left(\left|k_{0}\right|\right), \\
\Lambda_{12}=2 \pi \delta\left(K^{2}-m^{2}\right)\left(\Theta\left(-k_{0}\right)+n_{B}\left(\left|k_{0}\right|\right)\right), \\
\Lambda_{21}\left(k_{0}\right)=\Lambda_{12}\left(-k_{0}\right), \\
\Lambda_{22}=\left(\Lambda_{11}\right)^{*} .
\end{gathered}
$$

It can be seen, that if $m \gg T$ one can consider that only the 11 part is different from 0 . This is so because particles of type 2 are only inside loops, so there will always be a $\Lambda_{12} \Lambda_{21}$ term, that will be 0 because $\Theta\left(-k_{0}\right) \Theta\left(k_{0}\right)=0$.

\section{Hydrogen atom at $T \sim m \alpha^{2}$}

If in the Bose-Einstein (Fermi-Dirac) distribution function the energy $p_{0}$ is much bigger than the temperature $T$, then it goes exponentially to 0 . In a EFT language, this means that the influence of the temperature on the higher energy degrees of freedom can be neglected. In our case, this means that the $T=0$ pNRQED (2.2) can be taken as the starting point for the computation if $m \alpha \gg T$ and the error we are making is exponentially small (note that in this situation $e^{-m \alpha / T} \sim 10^{-59}$ ). 
Thermal effects will come from the degrees of freedom of (2.2). The relevant diagrams are the ones shown in fig. 2. Notice, that temperature does not affect vacuum polarisation because if $m \gg T$ there are no electrons in the thermal bath. The results from this computation are

$$
\begin{gathered}
\delta E_{n}=\frac{\pi \alpha}{3 m \beta^{2}}+e^{2} \lim _{q \rightarrow E_{n}} \sum_{m}\left\langle n\left|v^{i}\right| m\right\rangle \mathfrak{I} I_{i j}\left(q-E_{m}\right)\left\langle m\left|v^{j}\right| n\right\rangle, \\
\delta \Gamma_{n}=2 e^{2} \sum_{r}\left\langle n\left|v^{i}\right| r\right\rangle \Re I_{i j}\left(E_{n}-E_{r}\right)\left\langle r\left|v^{j}\right| n\right\rangle .
\end{gathered}
$$

Where,

$$
\begin{gathered}
\mathfrak{I}_{i j}(p)=\frac{2}{3} \frac{\delta_{i j} p}{(2 \pi)^{2}}\left(\log \left(\frac{2 \pi}{\beta|p|}\right)+\Re \psi\left(\frac{i \beta|p|}{2 \pi}\right)\right), \\
\Re I_{i j}(p)=\frac{\delta_{i j}}{6 \pi} \frac{|p|}{e^{\beta|p|}-1} .
\end{gathered}
$$

These results are also obtained considering the Stark effect induced by a blackbody radiation [13], and they have been successfully compared with experiments. However, the use of EFT makes the computation more systematic and one is more aware about the approximations being made.

\section{Hydrogen atom at $T \sim m \alpha$.}

Now, the Wilson coefficients of pNRQED are affected by temperature. So, the matching between NRQED and PNRQED have to be redone taking this into account. Moreover, whenever a computation is done in $\mathrm{PNRQED}$ for this temperature one has to expand the distribution function in order to be consistent with the EFT framework.

$$
\frac{1}{e^{\beta k}-1}=\frac{1}{\beta k}-\frac{1}{2}+\ldots
$$

But, this can induce ultraviolet divergences. Actually, this is what happens, but it is not a problem, as there is also a infrared divergence in the matching between NRQED and pNRQED.

After doing the matching and a field redefinition,

$$
\begin{gathered}
\delta L(T)=\int d^{3} x\left[-\frac{\pi \alpha}{6 m^{3} \beta^{2}} \vec{\nabla} \psi^{\dagger} \vec{\nabla} \psi+\left(\frac{\alpha \pi}{3 m \beta^{2}}\right) \psi^{\dagger} \psi\right]+ \\
+\int d^{3} x_{1} d^{3} x_{2} N^{\dagger} N\left(t, x_{2}\right)\left(-\frac{4 Z \alpha^{2}}{3 m^{2}}\left(\log \left(\frac{\beta \mu}{2 \pi}\right)+\gamma-\log 2+\frac{5}{6}\right) \times\right. \\
\left.\times \delta^{3}\left(x_{1}-x_{2}\right)\right) \psi^{\dagger} \psi\left(t, x_{1}\right) .
\end{gathered}
$$

Everything can be encoded in a mass redefinition and a change in the coefficient of the Darwin term (one of the fine-structure effects studied in atomic physics). This have to be summed with the $T=0$ lagrangian (2.2). We pointed out before that in (2.2) $c_{D}$ has a renormalization scale dependency, it happens that this dependency cancels out with the one in (4.2), so at the end it is a lagrangian that does not have any $\mu$ dependency, so we expect to have no ultraviolet divergences in contributions from the ultrasoft degrees of freedom.

Once we arrive to $P N R Q E D_{T}$ lagrangian, the relevant diagrams are again the ones of fig. 2 . At $T=0$ the second diagram have an ultraviolet divergence, but it happens that the $T=0$ plus the finite $T$ part is ultraviolet finite. 


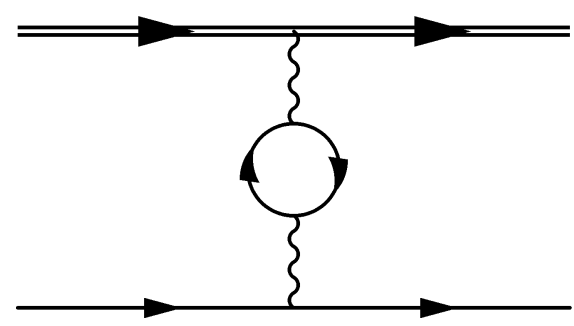

Figure 3: Vacuum polarisation effect to the potential

The results for this section are

$$
\begin{gathered}
\delta E_{n}=\frac{\alpha \pi}{3 m \beta^{2}}-\frac{\pi \alpha^{3}}{6 m \beta^{2} n^{2}}+\frac{2 \alpha}{3 \pi m^{2}} \sum_{r}\left|\left\langle n\left|p^{i}\right| r\right\rangle\right|^{2}\left(E_{n}-E_{r}\right)\left(\log \left(\frac{2 \pi}{\beta\left|E_{n}-E_{r}\right|}\right)-\gamma\right), \\
\delta \Gamma_{n}=\frac{4 Z^{2} \alpha^{3}}{3 \beta n^{2}}+\frac{2 \alpha}{3 m^{2}} \sum_{r}\left|\left\langle n\left|p^{i}\right| r\right\rangle\right|^{2}\left|E_{n}-E_{r}\right| .
\end{gathered}
$$

The limit of small temperature of these results are compatibles with the ones of $T \sim m \alpha^{2}$ (3.1,3.2), in the limit of high temperature. This is a crosscheck of the consistency of this method.

\section{Hydrogen atom at $T \sim m$.}

This situation is qualitatively and quantitatively very different from the previous ones. Now there are also electrons in the thermal bath, so the doubling of degrees of freedom have to be taken into account. Also, we can no longer neglect vacuum polarisation.

There are a lot of diagrams to be considered, but in a intuitive way the first place where one would look for crucial changes is the potential. In order to take into account the effect of vacuum polarisation in the potential the diagram of fig. 3 has to be computed.

If the temperature is high enough, the vacuum polarisation needs to be resummed (HTL resummation [12]). The potential that results from this is [14]

$$
V(r)=-\frac{Z \alpha e^{-m_{D} r}}{r}+\frac{i Z \alpha m_{d}^{3}}{\pi m_{D}^{2}} \phi\left(m_{D} r\right)
$$

$m_{D}$ and $m_{d}$ are functions of $m / T$.

- For $m / T \rightarrow 0, m_{D} \propto e T$ and $m_{d} \propto\left(T m_{D}^{2}\right)^{1 / 3}$.

- For $m / T \rightarrow \infty, m_{D} \propto \alpha m(m T)^{1 / 2} e^{-m / T}$ and $m_{d} \propto \alpha\left(m T^{2}\right)^{2 / 3} e^{-(2 m) /(3 T)}$.

In this potential there are two elements that can destroy the bound state. First, the screening that is due to $m_{D}$. This is what turns the real part into a Yukawa like potential (screening). But, there is also a imaginary part that will strongly increase the decay width. In the first studies of this problem only screening was taken into account [1], but our calculation, together with the calculations carried out recently, show that in fact the imaginary part is more relevant $[14,5,6]$.

This potential allow us to estimate the dissociation temperature. We mean by dissociation the temperature when the bound state disappears in a thermal bath of photons and electrons, and it is 


\begin{tabular}{|c|c|c|c|}
\hline $\mathrm{n}$ & $T_{d}(\mathrm{keV})$ & $m_{D}(\mathrm{keV})$ & $m_{d}(\mathrm{keV})$ \\
\hline 1 & 60.4 & 0.703 & 3.73 \\
2 & 50.1 & 0.284 & 1.86 \\
3 & 45.6 & 0.167 & 1.24 \\
4 & 42.9 & 0.114 & 0.932 \\
5 & 40.9 & 0.0842 & 0.746 \\
\hline
\end{tabular}

Table 1: Dissociation temperatures $T_{d}$ for hydrogen atom in the five lower energies states

not related to a hydrogen gas ionization temperature. This temperature can be approximated by the one when the decay width is of the order of the binding energy. In the potential, when the real part is of the order of the imaginary part. In our case $1 / r \sim m_{d}$.

The same can be done with heavy quarkonium, for $\Upsilon(1 S) T_{d} \sim 500 \mathrm{MeV}$, which is compatible with other studies and lattice results [15].For $J / \psi T_{d} \sim 200 \mathrm{MeV}$, it seems that charmonium's dissociation temperature is not hight enough for perturbation theory to be valid.

Since the dissociation temperature is found when $T \gg 1 / r \sim m_{d} \gg m_{D}$, some more steps can be done analytically.

$$
\begin{gathered}
\delta E_{n}=-\frac{\alpha m_{D}(Z-1)^{2}}{2}-\frac{Z \alpha m_{D}^{2}}{2}\langle n|r| n\rangle, \\
\delta \Gamma_{n}=2(Z-1)^{2} \alpha \sqrt{\frac{2}{\pi \beta^{3} m}}-\frac{2 Z \alpha m_{d}^{3}}{3 \pi}\left\langle n\left|r^{2}\left(\log \left(m_{D} r\right)+\gamma-4 / 3\right)\right| n\right\rangle .
\end{gathered}
$$

This yields $T_{d} \sim e^{4 / 3}(\log (1 / e))^{-1 / 3} m$. This result also holds for heavy quarkonium.

\section{Conclusions}

One of the aims of this work was to test the usefulness of EFT for this kind of problems. We have checked that the factorisation of scales still holds (which was no obvious due to Boltzmann factors) and that this approach gives consistent results. In particular, the limit $T \rightarrow 0$ in $T \sim m \alpha$ is the same as the limit $T \rightarrow \infty$ for $T \sim m \alpha^{2}$, and we have also found consistency in the cancellation of ultraviolet and infrared divergences for $T \sim m \alpha$. For $T_{d} \gg T$ we have reached a level of accuracy quite important (corrections of order $\left.m \alpha^{5}\right)(3.1,3.2)$.

For higher temperatures, we have found that the imaginary part of the potential is more important than screening, confirming the results of [14]. We have also been able to compute $T_{d}$ for the bound states of hydrogen atom, giving also estimates for heavy quarkonium. Finally, we have predicted $T_{d} \sim e^{4 / 3}(\log (1 / e))^{-1 / 3} m$.

\section{Acknowledgments}

I thank Joan Soto for the carefull reading of the manuscript.I also acknowledge financial support from the RTN Flavianet NRTN-CT-2006-035-482 (EU), the FPA 2007-60275/ and CPAN CSD2007-00042 grants (Spain), and the 2005SGR00564 CIRIT grant (Catalonia). I have also been supported by MICCIN FPU (Spain). 


\section{References}

[1] T. Matsui and H. Satz, Phys. Lett. B 178 (1986) 416.

[2] NA50 collaboration, Eur. Phys. J. C39 (2005), 335-345.

[3] PHENIX collaboration, H. Pereira da Costa, Nucl. Phys. A77 (2006), 747-750

[4] H. Satz, J. Phys. G 32 R25 (2006)

[5] M. A. Escobedo and J. Soto, Phys. Rev. A 78, 032520 (2008) [arXiv:0804.0691 [hep-ph]]

[6] N. Brambilla, J. Ghiglieri, A. Vairo and P. Petreczky, Phys. Rev. D 78 (2008) 014017 [arXiv:0804.0993 [hep-ph]].

[7] N. Brambilla et al. [Quarkonium Working Group], arXiv:hep-ph/0412158.

[8] W. E. Caswell and G. P. Lepage, Phys. Lett. B 167 (1986) 437.

[9] A. Pineda and J. Soto, Nucl. Phys. Proc. Suppl. 64 (1998) 428 [arXiv:hep-ph/9707481].

[10] A. Pineda and J. Soto, Phys. Lett. B 420 (1998) 391 [arXiv:hep-ph/9711292].

[11] N. Brambilla, These proceedings.

[12] M. Le Bellac, Thermal Field Theory, Cambridge University Press

[13] J. W. Farley and W. H. Wing, Phys. Rev. A 23, 2397 (1981)

[14] M. Laine, O. Philipsen, P. Romatschke and M. Tassler, JHEP 0703 (2007) 054 [arXiv:hep-ph/0611300].

[15] M. Laine, JHEP 0705 (2007) 028 [arXiv:0704.1720 [hep-ph]]. 